\title{
Retinal function in relation to improved glycaemic control in type 1 diabetes
}

\author{
S. K. Holfort • K. Nørgaard • G. R. Jackson • \\ E. Hommel • S. Madsbad • I. C. Munch • K. Klemp • \\ B. Sander • M. Larsen
}

Received: 23 November 2010 / Accepted: 17 March 2011 / Published online: 26 April 2011

(C) Springer-Verlag 2011

\begin{abstract}
Aims/hypothesis To study long-term changes in retinal function in response to sustained glycaemia reduction in participants with type 1 diabetes.

Methods Prospective study using objective measures of retinal function in 17 participants with type 1 diabetes mellitus and minimal to moderate retinopathy who switched from conventional subcutaneous injection to continuous subcutaneous infusion of insulin (CSII).

Results Glycated haemoglobin $\mathrm{HbA}_{1 \mathrm{c}}$ gradually decreased from $9.1 \%$ at baseline before CSII to $7.4 \%$ after 1 year on CSII. Glycaemia was markedly reduced within 1 week after initiation of CSII and remained stable thereafter. Dark adaptation and retinal electroretinographic function at 1, 4 and 16 weeks after initiation of CSII were comparable with baseline values, whereas a significant improvement in rod
\end{abstract}

S. K. Holfort $(\bowtie) \cdot$ I. C. Munch $\cdot$ K. Klemp $\cdot$ B. Sander •

M. Larsen

Department of Ophthalmology, Glostrup Hospital,

Nordre Ringvej 57,

2600 Glostrup, Denmark

e-mail: STKRHO02@glo.regionh.dk

K. Nørgaard $\cdot$ S. Madsbad

Department of Endocrinology, Hvidovre Hospital,

Hvidovre, Denmark

G. R. Jackson

Penn State Hershey Eye Center, Penn State College of Medicine,

Hershey, PA, USA

E. Hommel

Steno Diabetes Center,

Copenhagen, Denmark

M. Larsen

Kennedy Center, National Eye Clinic,

Glostrup, Denmark photoreceptor dark adaptation and dark-adapted b-wave amplitudes were seen after 52 weeks (time to rod-cone break $-25 \%[p<0.0001]$, time to a standardised rod intercept $-13 \%[p<0.0001]$, dark-adapted rod b-wave fullfield amplitude $+15 \%[p=0.0125]$, standard combined rodcone $\mathrm{b}$-wave amplitude $+8 \%[p=0.049])$. No detectable change was observed in cone adaptation, electroretinographic cone function or retinopathy.

Conclusions/interpretation After initiation of CSII, the retinal visual pathway of the rods improved with a delay of more than 4 months, over a time scale comparable with the duration of the diabetic retinopathy early worsening response to sustained glycaemia reduction. This indicates that glycaemia has a long-term effect on the disposition of functional capacity in the retinal visual pathway of rod photoreceptors, the cells that appear to be driving the development of diabetic retinopathy.

Keywords Dark adaptation - Diabetic retinopathy

Early worsening $\cdot$ Electrophysiology

\author{
Abbrevations \\ CSII Continuous subcutaneous insulin infusion \\ ETDRS Early Treatment Diabetic Retinopathy Study \\ ffERG Full-field electroretinography \\ ISCEV International Society for Clinical \\ Electrophysiology of Vision \\ mfERG Multifocal electroretinography
}

\section{Introduction}

Diabetic retinopathy is a microvascular disease of the retina in people with diabetes that presents with a range of 
localised, ophthalmoscopically visible lesions including intraretinal haemorrhage, microaneurysm, axonal thickening, dilated and tortuous vessels, exudative oedema and proliferation of new vessels $[1,2]$. The lesions are associated with endothelial dysfunction, pericyte and endothelial cell loss, and proliferation of endothelial cells. On a microscopic level, there are also considerable abnormalities to be seen in and around neuronal and glial cells of the retina and some functional abnormalities, even before the appearance of ophthalmoscopic retinopathy, but as in early and moderate levels of diabetic retinopathy the dysfunction seen in the absence of retinopathy is subclinical [3]. It remains to be determined whether diabetic retinopathy is primarily a neuroglial or microvascular disease [3-9].

On a population level, diabetic retinopathy increases in prevalence and severity with increasing duration of diabetes [2]. On the level of the individual with diabetes, however, retinopathy can wax and wane in severity and even improve significantly without treatment of the eye $[10,11]$.

The principal underlying cause of retinopathy appears to be abnormal glycaemia, and over periods longer than approximately 2 years, hyperglycaemia is a strong risk factor for progression to more severe levels of retinopathy [12]. In the short run, however, the relation is more complex, and several studies have shown that therapeutically induced hyperglycaemia reduction leads to worsening of retinopathy within the first year [10, 13-15]. This early worsening phenomenon has eluded previous attempts at explanation. Functional abnormalities in the retina have been found by electroretinography [5, 16-19], dark adaptometry [20] and contrast sensitivity testing [7,21], even in the absence of diabetic retinopathy, but their cause is not understood. There is no well-defined link between functional abnormalities and tissue damage, and it is largely unknown whether the former are reversible or irreversible. In the present study we examined retinal function over 1 year to characterise the time course of retinal adaptation to relative hypoglycaemia after participants with type 1 diabetes had shifted from conventional subcutaneous insulin injection to continuous subcutaneous insulin infusion (CSII).

\section{Methods}

Participants The study included 17 eyes in 17 participants with type 1 diabetes mellitus, and minimal to moderate diabetic retinopathy (Early Treatment Diabetic Retinopathy Study [ETDRS] levels 10-43). All participants were recruited from the Steno Diabetes Center and the Hvidovre Hospital. The study included participants with $\mathrm{HbA}_{1 \mathrm{c}} \geq 8 \%$ at baseline and no history of ocular surgery or laser treatment. Within 16 weeks of CSII initiation the partic- ipant's $\mathrm{HbA}_{1 \mathrm{c}}$ was required to be $\leq 7.5 \%$ or be reduced by $\geq 1 \%$ or the participant was excluded. Further exclusion criteria included haemoglobin, sodium, potassium or creatinine outside the normal range of the clinical biochemistry laboratory of the Glostrup Hospital, microalbuminuria $(>30 \mathrm{mg} / \mathrm{l})$, cataract, a family history of glaucoma, intraocular pressure by applanation tonometry $>21 \mathrm{mmHg}$, arterial blood pressure higher than $140 / 90 \mathrm{mmHg}$, clinically significant macular oedema and proliferative diabetic retinopathy. Of the 17 participants, six used a single antihypertensive agent (ACE inhibitor), three used a cholesterol-lowering agent, two used an anticoagulant, one used a thiazide diuretic and one used an anti-thyroid and an antihistamine agent. Ethical approval was granted by the Danish National Committee on Biomedical Research Ethics. Informed consent was obtained from the participants before commencing the study, which was conducted in accordance with the Helsinki Declaration.

Study procedures Participants initially underwent an ophthalmological examination and fundus photography including pupil dilation to a diameter $\geq 7 \mathrm{~mm}$ using topical phenylephrine hydrochloride $10 \%$ and tropicamide $1 \%$. The thickness and reflectivity profile of the retina were examined by transfoveal optical coherence tomography. Grading of retinopathy was based on an abbreviated version of the ETDRS protocol using one non-stereoscopic $50^{\circ}$ colour fundus photograph centred on the fovea and one centred on the optic disc. The grading was performed by two retinal specialists, who were blinded with regard to participant identity, chronological order of examinations, and test outcomes. The eye with the lower degree of retinopathy was chosen as the study eye.

Participants were scheduled to five visits: 1 week before initiation of CSII and 1, 4, 16 and 52 weeks after initiation of CSII. Participants were instructed to measure their capillary blood glucose four times per day (before breakfast, before lunch, before dinner and before bedtime) for 3 days before the baseline visit and for 7 days before the 1 week visit. Subsequently, blood glucose was measured four times per day for 3 days before the visit and every second week between visits. Participants were asked to follow their usual dietary routine and came non-fasting to the eye examination sessions. They were requested to adjust their insulin treatment so that glycaemia was no greater than $15 \mathrm{mmol} / \mathrm{l}$ during the sessions in order to achieve similar glucose levels during testing of retinal function. At every session venous haemoglobin, sodium, potassium, creatinine, $\mathrm{HbA}_{1 \mathrm{c}}$, blood pressure and visual acuity were determined and the participants underwent the following examination programme of the study eye: pupil dilation to a diameter of $\geq 7 \mathrm{~mm}$ using phenylephrine hydrochloride $10 \%$ and tropicamide $1 \%$ followed by dark 
adaptometry, fundus photography, biomicroscopy examination and optical coherence tomography. Finally, after a 30 min rest, the participants underwent multifocal electroretinography (mfERG) and full-field electroretinography (ffERG). Capillary blood glucose was measured before and after dark adaptometry, mfERG and ffERG. One participant was unable to reliably perform dark adaptometry at baseline and therefore did not undergo this procedure at the subsequent visits and was excluded from the analysis of dark adaptometry data.

Methods Fundus photography (FF 450plus Fundus Camera; Carl Zeiss Meditec AG, VISUPAC, Jena, Germany) was made after pupil dilation, as was horizontal transfoveal optical coherence tomography (Cirrus; Carl Zeiss Meditec, CA, USA). Capillary blood glucose was measured using a reagent strip and reading device (Precision Xceed; Medisense Products, Abbott Laboratories, Witney, UK).

Dark adaptation was measured using a computerised dark adaptometer (AdaptDx; Apeliotus Technologies, Atlanta, GA, USA) [22]. The fellow eye was occluded with an eye patch. An infrared camera located behind the fixation light was used to continuously monitor the test eye. The operator centred the participant interactively with reference to a reticule overlaid on a computer display of the test eye. The participant's test eye was bleached by exposure to a white photoflash $(0.25 \mathrm{~ms}$ duration, $6.38 \mathrm{log}$ scotopic td s${ }^{-1}$ intensity) while the participant focused on the fixation light. The flash was passed through a diffuser and a $4^{\circ}$ diameter aperture centred $5^{\circ}$ below the point of fixation. Sensitivity measurements began immediately after bleach offset. The participant focused on the fixation light and responded to each stimulus seen by manually pressing a button. The light stimulus was a $1.7^{\circ}$ circular test spot centred $5^{\circ}$ below the point of fixation. To focus on rodmediated function, the stimulus wavelength was at $500 \mathrm{~nm}$, near the peak of the rod spectral sensitivity function. Starting at a relatively high intensity $\left(50\right.$ scotopic $\left.\mathrm{cd} / \mathrm{m}^{2}\right)$, stimuli were presented for $200 \mathrm{~ms}$ every $2-3 \mathrm{~s}$. If the participant did not respond by pressing a button within $2 \mathrm{~s}$ of stimulus onset, the intensity remained unchanged on successive stimulus presentations until the participant responded. If the participant responded to the stimulus, the intensity was decreased for each successive presentation in steps of $0.3 \log$ units until the participant stopped responding. When the participant stopped responding, the intensity was increased $0.1 \mathrm{log}$ units for each successive presentation until the participant responded again. This intensity was taken to represent a threshold for a given interval after bleaching. Subsequent threshold assessments started with a stimulus intensity $0.2 \log$ units brighter than the previous threshold measurement. The participant had a 10 s rest between threshold measurements. Sensitivity recovery was measured for $20 \mathrm{~min}$ or until the participant was fully recovered through the second component of rodmediated dark adaptation.

The speed of dark adaptation was characterised using two methods. The first was to fit a single-exponential, onelinear component model to the data. This model allows for the estimate of the cone recovery time constant, rod cone break and slope of the second component of rod-mediated dark adaptation [23]. The second method was to estimate the time required to reach a rod-mediated criterion sensitivity level.

This criterion sensitivity was set at $5 \times 10^{-3}$ scotopic cd/ $\mathrm{m}^{2}$ (4.0 log units), a sensitivity level that is achieved late in the second component of rod-mediated dark adaptation [22, 24]. The rod intercept was estimated by linear interpolation of the relevant segment of the rod adaptation curve. The rod intercept is useful to describe the speed of rod-mediated dark adaptation because it is robust to the shape of the dark adaptation curve, which changes with increased disease severity.

Full-field ERG was recorded using a Nicolet GS 2000 Ganzfeld stimulator and amplifier (Nicolet, Madison, WI, USA). An optimally fitting Burian-Allen bipolar contact lens was identified at the baseline recording and used again for the subsequent recordings. A ground electrode was attached to the forehead. The following variables were determined based on International Society for Clinical Electrophysiology of Vision (ISCEV) standards [25]: dark-adapted rod response, dark-adapted standard combined rod-cone response, dark-adapted oscillatory potentials, light-adapted single cone response and light-adapted $30 \mathrm{~Hz}$ flicker response. In addition, dark-adapted $30 \mathrm{~Hz}$ flicker $\left(3.93 \mathrm{~cd} \mathrm{~s}^{-1} \mathrm{~m}^{-2}\right.$ ) was determined. Topical oxybuprocaine $0.4 \% \mathrm{mg} / \mathrm{ml}$ was used to anaesthetise the cornea.

Multifocal electroretinography in the study eye was made using an optimally sized Burian-Allen bipolar contact lens electrode (Veris IR Illuminating Electrode; EDI, San Mateo, CA, USA) with two built-in infrared light sources for fundus illumination. The fellow eye was occluded. A ground electrode was attached to the forehead. Visual stimuli were displayed on a 1.5-in. stimulator/fundus camera (Veris; EDI), permitting optimal correction of refraction without changing the size of the stimulus elements and ensuring fixation by real-time infrared observer viewing of the fundus. An array of 103 eccentricity-scaled hexagons was displayed at a frame rate of $75 \mathrm{~Hz}$. Responses were band-pass filtered outside 10 to $300 \mathrm{~Hz}$, amplified at gain 105 and sampled every $0.833 \mathrm{~ms}$. A standard m-sequence length was used with $m=15$, resulting in a total recording time of $7.17 \mathrm{~min}$, divided into eight short segments for participant comfort. If loss of fixation or an artefact was observed the affected segment 
was discarded and re-recorded. The luminance of white stimuli was $200 \mathrm{vs} 2 \mathrm{~cd} / \mathrm{m}^{2}$ or less for black stimuli. The surround luminance was set to $50 \%$ of the bright test luminance (i.e. $100 \mathrm{~cd} / \mathrm{m}^{2}$ ). The examination was made in ambient room lighting. Stimulus luminance was calibrated using an autocalibrator. The stimulus grid was calibrated using a grid calibrator (Veris; EDI). The recording protocol complied with the ISCEV guidelines for basic mfERG [26].

Analysis Dark adaptation functions were plotted using Igor Pro (Wavemetrics, Portland, OR, USA). Nonlinear regression was used to estimate the speed of dark adaptation using a single-exponential one-linear model, which uses an unbiased method to calculate estimates of the cone sensitivity plateau value, cone sensitivity recovery time constant, rod-cone break time and rod sensitivity recovery slope [23]. In addition, the rod intercept value was determined as described above, using interpolation, as a shape-invariant method of characterisation of the rate of rod-mediated dark adaptation $[22,24]$. Cases where no rod-cone break or no rod intercept were obtained were given the value $20 \mathrm{~min}$, which is the maximum test time for the adaptometer. A group of non-diabetic participants with healthy eyes aged $73.1 \pm 4.8$ years that has been described before was used for reference [22]. Dark adaptation was classified as normal if the rod intercept was within the mean $\pm 2 \mathrm{SD}$ of the reference participants and impaired if longer than the upper limit of this interval (12.5 min).

Full-field electroretinography was analysed as follows. The a-wave amplitude was measured from the baseline to the trough of the first negative deflection. In the absence of an awave, the b-wave amplitude was measured from the baseline to the peak. In the presence of an a-wave, the b-wave amplitude was measured from the trough of the a-wave to the peak of the b-wave. The implicit times of the a- and bwaves were measured from the onset of the light stimulus to the peak of the amplitude of each response component. The amplitude of the $30 \mathrm{~Hz}$ flicker response was measured from the trough to the peak and the implicit time was measured from the onset of the light stimulus to the amplitude peak.

Multifocal electroretinography analysis began with two iterations of artefact rejection applied to the raw data without spatial smoothing. The first- and second-order kernels were derived and implicit times and amplitudes of the components $\mathrm{N} 1$ (first negative), P1 (first positive) and $\mathrm{N} 2$ (second negative) were measured. The N1 amplitude was measured from the starting baseline to the base of the $\mathrm{N} 1$ trough, the P1 response amplitude from the N1 trough to the P1 peak and the N2 amplitude was measured from the P1 peak to the N2 trough. Average responses were calculated for the summed values of all hexagons, for the seven most central hexagons and for the surrounding rings.

Statistical evaluation Mean optical coherence tomography thickness of the fields of the interpolated retinal thickness map and mean capillary glucose levels during examinations were analysed using mixed model analysis to test for changes between baseline and each of the follow-up visits. Glucose profiles before visits were summed and a mean value calculated and used to represent glucose levels before visits. Dark adaptometry data were analysed by calculating the differences between the baseline visit and the final visit (52 weeks). Variables included the cone plateau, the time to reach the cone plateau, the slope of the rod adaptation curve, the time to the rod-cone break and the time to the rod intercept. Differences were analysed by the one-sample $t$ test. We found the measurements to be unsuitable for determination of the time to the cone plateau because there were too few measurements to define it and hence this variable was rejected during analysis. Linear regression was used to analyse relations between differences in $\mathrm{HbA}_{1 \mathrm{c}}$ and dark adaptation kinetics between the baseline and the final visit.

Full-field ERG data were analysed by comparing the changes in ffERG amplitude and implicit time. Change was defined as the difference between ERG recordings at the final visit (52 weeks) and baseline. Data were analysed using

Table 1 Glycaemia and change in retinopathy grading after initiation of continuous subcutaneous insulin infusion in 17 eyes in 17 patients with diabetes

\begin{tabular}{lcccc}
\hline Time after CSII (weeks) & $\mathrm{HbA}_{1 \mathrm{c}}(\%)$ & Retinopathy improved & Retinopathy unchanged & Retinopathy worsened \\
\hline Baseline & $9.1 \pm 2$ & - & 17 & - \\
1 & $8.7 \pm 2$ & 3 & 12 & 2 \\
4 & $8.2 \pm 1$ & 2 & 13 & 2 \\
16 & $7.5 \pm 2$ & 2 & 9 & 4 \\
52 & $7.4 \pm 1$ & 4 & 9 & \\
\hline
\end{tabular}

Values are mean \pm SD or number of eyes (one study eye per patient)

Retinopathy grading levels according to the EDTRS, improvement/worsening defined as decrease or increase, respectively, of one or more retinopathy levels 


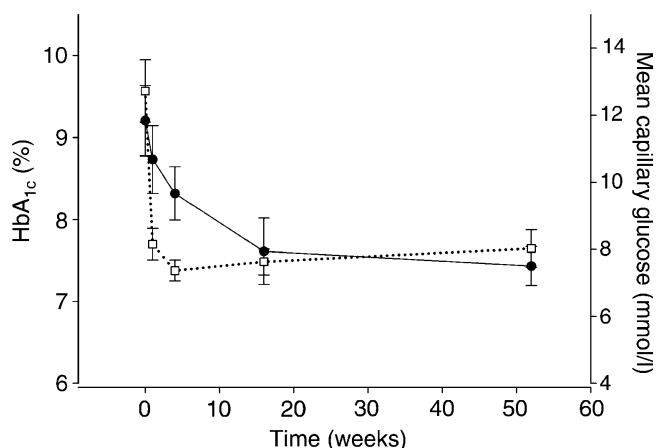

Fig. 1 Mean $\mathrm{HbA}_{1 \mathrm{c}}$ (black circles) measured at visit $(n=17)$ and mean capillary blood glucose (white squares) measured by patients at home $(n=15)$ in the days leading up to the visit. Patients were examined before (time $=0$ ) and $1,4,16$ and 52 weeks after initiation of continuous subcutaneous insulin infusion. Error bars represent standard error of the mean

the one-sample paired $t$ test. Linear regression was used to analyse relations between level of $\mathrm{HbA}_{1 \mathrm{c}}$ and $\mathrm{ERG}$ variables.

Multifocal ERG first- and second-order data were analysed by comparing the difference in amplitudes and implicit times between the baseline and the final visit (52 weeks). The multifocal ERG was analysed as a summed response and as a centre vs periphery by analysis of the seven most central hexagons against each of the peripheral rings of hexagons.

Finally, dark adaptometry-, ffERG- and mfERG data were analysed by mixed model analysis to test for changes between baseline and each of the subsequent visits. Adjustment was made for glycaemia at the time of examination, diabetes duration and $\mathrm{HbA}_{1 \mathrm{c}}$.

The level of statistical significance was set at $p<0.05$. Statistical analysis was performed using the SAS 9.1 software for Windows (SAS Institute, Cary, NC, USA).

\section{Results}

The 17 participants (6 men and 11 women) had a mean age of $38.2 \pm 11.8$ years, a mean duration of diabetes of $22.4 \pm$
10 years and a mean $\mathrm{HbA}_{1 \mathrm{c}}$ of $9.1 \%$ at baseline (Table 1 ). Participant-performed capillary glucose measurements and their accompanying $\mathrm{HbA}_{1 \mathrm{c}}$ curves demonstrate that a stable reduction of glycaemia had been achieved already after 1 week (Fig. 1).

Retinopathy level was unchanged between baseline and 52 weeks in nine eyes, decreased by one level in four eyes and increased by one level in four eyes (Table 1). No eye developed macular oedema or proliferative retinopathy and there was no significant change in the mean retinal thickness during the study. Best-corrected visual acuity was Snellen 1.0 or better in all participants at all visits.

Rod-dark adaptation was comparable to baseline up to 16 weeks after beginning CSII, but was considerably faster after 52 weeks (Table 2). The time to the rod-cone break had shortened by $25 \%$ after 52 weeks ( $95 \%$ CI $14-35 \%$; $p=$ 0.0009 ; Fig. 2) while the time to the rod intercept had shortened by $13 \%(95 \%$ CI $1-15 \%$; $p<0.0001$; Table 2$)$. There was no detectable correlation between the change in $\mathrm{HbA}_{1 \mathrm{c}}$ and the change in rod-cone break time or rod intercept between baseline and 52 weeks. In a mixed model analysis dark adaptation was only different from baseline after 52 weeks (time to rod-cone break $p<0.0001$, time to rod intercept $p<0.0001)$. Cone adaptation did not change significantly during the study.

The dark-adapted rod response of the ffERG was stable up to 16 weeks after initiation of CSII but showed an increase in b-wave amplitude of $15 \%$ (95\% CI 3$26 \% ; p=0.02$ ) between baseline and 52 weeks (Table 3 and Fig. 3). Comparable trends in the a-wave of the standard combined rod-cone response $(+8 \%$; $95 \%$ CI 0 $17 \% ; p=0.08)$ and in $\mathrm{b}$-wave amplitude $(+8 \% ; 95 \%$ CI 0 $16 \%$; $p=0.1$; Table 3 ) did not reach statistical significance. No detectable change in amplitude was found for oscillatory potentials, dark-adapted $30 \mathrm{~Hz}$ flicker, lightadapted single cone or light-adapted $30 \mathrm{~Hz}$ flicker response. Changes in $\mathrm{HbA}_{1 \mathrm{c}}$ and ffERG variables could not be shown to be correlated. Mixed model analysis

Table 2 Dark adaptation in participants with type 1 diabetes before and after initiation of continuous subcutaneous insulin infusion

\begin{tabular}{lcccc}
\hline Time after CSII (weeks) & $\begin{array}{l}\text { Final cone } \\
\text { threshold }(\log )\end{array}$ & $\begin{array}{l}\text { Time to rod-cone } \\
\text { break (min) }\end{array}$ & $\begin{array}{l}\text { Time to rod } \\
\text { intercept (min) }\end{array}$ & $\begin{array}{l}\text { Rod sensitivity recovery } \\
\text { slope (log/min) }\end{array}$ \\
\hline Baseline & $2.9 \pm 0.2$ & $6.5 \pm 1.8$ & $10.5 \pm 2.1$ & $0.30 \pm 0.09$ \\
1 & $2.9 \pm 0.2$ & $6.1 \pm 0.9$ & $10.2 \pm 1.8$ & $0.30 \pm 0.07$ \\
4 & $3.0 \pm 0.3$ & $6.4 \pm 1.4$ & $10.2 \pm 1.9$ & $0.30 \pm 0.08$ \\
16 & $3.0 \pm 0.3$ & $6.4 \pm 1.9$ & $10.0 \pm 1.9$ & $0.31 \pm 0.08$ \\
52 & $2.9 \pm 0.2$ & $4.9 \pm 1.3^{\dagger}$ & $9.1 \pm 2.0^{\dagger}$ & $0.30 \pm 0.06$ \\
\hline
\end{tabular}

Values are mean $\pm \mathrm{SD}$

$n=16$ : one patient was unable to cooperate at dark adaptometry

${ }^{\dagger} p<0.0001$ compared with baseline 


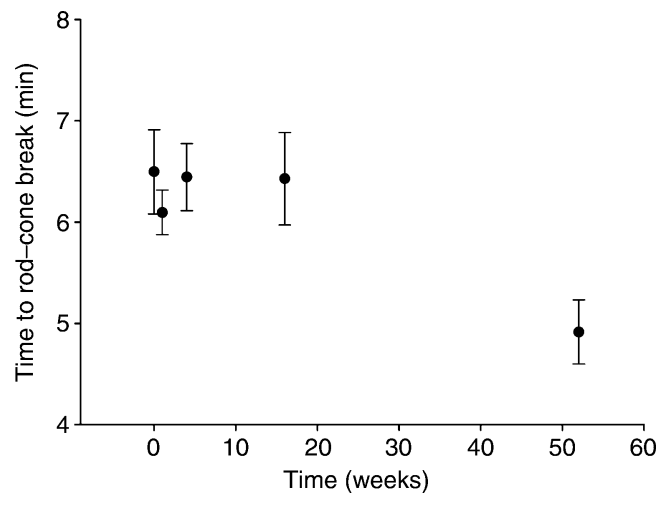

Fig. 2 Mean time to rod-cone break during dark adaptation in participants with type 1 diabetes before (time=0) and 1, 4, 16 and 52 weeks after initiation of continuous subcutaneous insulin infusion. Error bars represent standard error of the mean

showed that changes between baseline and final visit were significant for the dark-adapted rod response b-wave amplitude $(p=0.0125)$ and the $\mathrm{b}$-wave of the standard combined rod-cone response $(p=0.049)$ but not for the awave amplitude $(p=0.095)$. No significant changes in implicit times were found. Although the variation of the rod-cone break and the scotopic b-wave amplitude between baseline and 16 weeks did not reach significance, it is noteworthy that the two curves closely mirror each other throughout the study (Figs 2 and 3).

The first- and second-order amplitudes and implicit times of the summed response, the seven foveal hexagons and the peripheral ring of the mfERG did not show any significant change between the baseline and the final visit (Table 4). Mixed model analysis did not show significant changes between the baseline visit and the following visits for the summed response, the seven foveal hexagons or the peripheral rings. Adjustment for glycaemia during examination sessions, diabetes duration and $\mathrm{HbA}_{1 \mathrm{c}}$ did not affect the outcome of the mixed model analyses of dark adaptation, ffERG and mfERG.

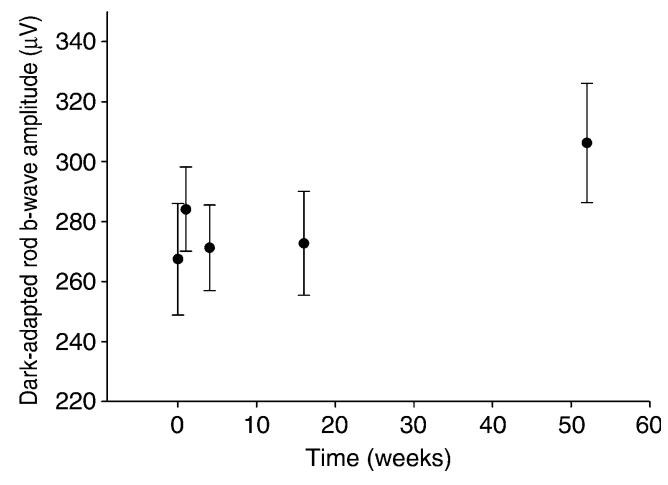

Fig. 3 Dark-adapted rod b-wave amplitude during ffERG in participants with type 1 diabetes $(n=17)$ before (time $=0)$ and 1,4 , 16 and 52 weeks after initiation of continuous subcutaneous insulin infusion. Error bars represent standard error of the mean

\section{Discussion}

Protracted retinal adaptation to reduced glycaemia has previously been observed in the form of a change in the rate of diabetic retinopathy progression. Within the first year improved glycaemia control in type 1 diabetes has been associated with an increase in cotton wool spots, intraretinal microvascular abnormalities, proliferative retinopathy, diabetic papillopathy and macular oedema $[10,14$, $15,27]$. In the DCCT, intensified insulin therapy was accompanied by a biphasic change in retinopathy progression, beginning with $0.5-1$ year of accelerated progression followed by decelerated progression from 1.5 years and onwards, compared with a control group that remained on conventional insulin therapy [10]. In the present study, we observed a change in retinal performance beginning no earlier than after 16 weeks of intensified insulin treatment, which resulted in improved neuronal signalling of ONbipolar cells and/or Müller cells in the middle layers of the retina and accelerated dark adaptation of the of the rod photoreceptors after 1 year. Thus, there is an interesting temporal relationship between simple and objective linear

Table 3 Electroretinographic amplitudes in type 1 diabetes before and after initiation of continuous subcutaneous insulin infusion

\begin{tabular}{|c|c|c|c|c|c|c|c|}
\hline \multirow{2}{*}{$\begin{array}{l}\text { Time after } \\
\text { initiation of } \\
\text { CSII (weeks) }\end{array}$} & \multicolumn{4}{|c|}{ Dark-adapted responses: amplitude $(\mu \mathrm{V})$} & \multicolumn{3}{|c|}{ Light-adapted responses: amplitude $(\mu \mathrm{V})$} \\
\hline & $\begin{array}{l}\text { Dark-adapted } \\
\text { rod }\end{array}$ & $\begin{array}{l}\text { Combined } \\
\text { rod-cone a-wave }\end{array}$ & $\begin{array}{l}\text { Combined } \\
\text { rod-cone b-wave }\end{array}$ & $30 \mathrm{~Hz}$ flicker & $\begin{array}{l}\text { Single cone } \\
\text { a-wave }\end{array}$ & $\begin{array}{l}\text { Single cone } \\
\text { b-wave }\end{array}$ & $30 \mathrm{~Hz}$ flicker \\
\hline Baseline & $268 \pm 77$ & $239 \pm 71$ & $472 \pm 116$ & $72 \pm 24$ & $39 \pm 12$ & $161 \pm 59$ & $129 \pm 43$ \\
\hline 1 & $284 \pm 58$ & $250 \pm 62$ & $497 \pm 91$ & $75 \pm 19$ & $38 \pm 14$ & $166 \pm 48$ & $127 \pm 38$ \\
\hline 4 & $271 \pm 59$ & $255 \pm 61$ & $497 \pm 103$ & $72 \pm 15$ & $43 \pm 16$ & $168 \pm 39$ & $133 \pm 39$ \\
\hline 16 & $273 \pm 72$ & $233 \pm 45$ & $480 \pm 69$ & $71 \pm 17$ & $40 \pm 11$ & $162 \pm 39$ & $120 \pm 29$ \\
\hline 52 & $307 \pm 81 \dagger$ & $258 \pm 59$ & $508 \pm 76+$ & $75 \pm 16$ & $42 \pm 16$ & $173 \pm 41$ & $132 \pm 39$ \\
\hline
\end{tabular}

Values are mean $\pm \mathrm{SD}$

${ }^{\dagger} p=0.0125,{ }^{*} p=0.049$ compared with baseline 
Table 4 Multifocal electroretinography amplitudes and implicit times in type 1 diabetes in relation to time after initiation of continuous subcutaneous insulin infusion

\begin{tabular}{|c|c|c|c|c|c|c|}
\hline \multirow{2}{*}{$\begin{array}{l}\text { Time after initiation } \\
\text { of CSII (weeks) }\end{array}$} & \multicolumn{3}{|c|}{ Amplitude $(\mu \mathrm{V})$} & \multicolumn{3}{|c|}{ Implicit time (ms) } \\
\hline & N1 & P1 & $\mathrm{N} 2$ & N1 & $\mathrm{P} 1$ & $\mathrm{~N} 2$ \\
\hline Baseline & $-7.7 \pm 2.5$ & $13.2 \pm 4.7$ & $-9.9 \pm 3.5$ & $15.9 \pm 0.8$ & $29.0 \pm 1.0$ & $43.1 \pm 1.1$ \\
\hline 1 & $-7.1 \pm 2.0$ & $12.2 \pm 3.6$ & $-9.1 \pm 2.9$ & $15.8 \pm 0.8$ & $28.5 \pm 1.5$ & $42.8 \pm 1.4$ \\
\hline 4 & $-7.6 \pm 2.0$ & $13.2 \pm 3.6$ & $-9.9 \pm 2.9$ & $15.7 \pm 0.8$ & $28.9 \pm 1.2$ & $43.2 \pm 1.3$ \\
\hline 16 & $-7.3 \pm 2.2$ & $12.6 \pm 3.5$ & $-9.5 \pm 2.4$ & $15.8 \pm 0.9$ & $29.0 \pm 1.4$ & $43.4 \pm 1.5$ \\
\hline 52 & $-8.3 \pm 2.2$ & $14.5 \pm 4.2$ & $-10.9 \pm 3.0$ & $15.9 \pm 1.0$ & $29.0 \pm 1.2$ & $43.6 \pm 1.6$ \\
\hline
\end{tabular}

Values are mean $\pm \mathrm{SD}$

No significant variations in multifocal electroretinography amplitudes and implicit times were observed over time

measures of retinal physiology and complex, noncontinuous morphological measures of retinopathy severity. In the present study progression of retinopathy was not observed; however, the participants did not have aggressive retinopathy at baseline and the abbreviated retinopathy grading protocol may have limited the sensitivity of the study.

Irreversible reduction of retinal performance by laser destruction of photoreceptors in the peripheral retina is an effective method of reducing loss of foveal function and preserving visual acuity in diabetic retinopathy. This effect suggests that modulation of retinal performance - in this case elimination of retinal function-influences the progression of diabetic retinopathy. It is therefore of interest to determine how the need or propensity of the retina to modulate its function after a change in glycaemia may affect microvascular integrity in the retina.

Early worsening of diabetic retinopathy tends not to occur to any clinically noteworthy degree in diabetic patients with minimal or no retinopathy [28, 29]. Patients with retinopathy progression in relation to glycaemia reduction generally have higher levels of diabetic retinopathy and poor prior metabolic control of long duration [10, $15,27]$. Consequently, the results of the present study cannot unreservedly be assumed to be representative for all patients with diabetes. Both baseline values and the response to CSII may vary between patients with different levels of retinopathy and different glycaemia characteristics.

Compared with non-diabetic participants, slower rod and cone function and reduced rod sensitivity have been demonstrated in patients with diabetes [5, 9, 16, 30, 31]. Such findings are difficult to interpret, however, because retinal performance fluctuates with glycaemia, [32-34], which is inherently unstable in diabetes. Specifically, an acute increase in glycaemia has been shown to accelerate light-adapted cone function, increase dark-adapted rod sensitivity and accelerate rod dark adaptation in patients with diabetes $[16,35,36]$. Furthermore, there is also an effect of participants' long-term glycaemia history [16, 35].

The shift in retinal performance seen in this study occurred with a delay of at least 4 months, during a period when glycaemia was stable. With only a single examination after the onset of improvement, it is not possible to determine whether a stable level of performance had been reached after 1 year. In fact, the lack of a correlation between the change in $\mathrm{HbA}_{1 \mathrm{c}}$ and the change in dark adaptation and ffERG characteristics may be explained by the absence of a steady state of the adaptation process. We have previously observed an adaptation phenomenon occurring on a comparable timescale following a change in ambient oxygen level [37], suggesting that there may be a common retinal mechanism of adaptation to a change in the supply of either of the two primary fuels of the retina, oxygen and glucose.

It is not known how a chronic change in glycaemia can induce a shift in retinal performance. There are noteworthy characteristics of the retina, however, that may offer some clues. The outer layers of the retina and the entire fovea are avascular, hypoxic and lactate-producing, especially in the dark-adapted state [38-40]. The retina is at its highest energy consumption in the dark-adapted state, rod photoreceptors having a higher metabolic rate than any other cell in the human body [41, 42]. We postulate the existence of a retinal mechanism that balances aerobic and anaerobic metabolism so that performance and sustainability are optimised for a given balance between oxygenaemia and glycaemia. This hypothetical mechanism would need to be reset when glycaemia is reduced relative to oxygenaemia by intensified insulin treatment $[16,35,36]$. In this context Müller cells, contributing to the b-wave of the ffERG responses [43], play an important role in the normal function of the retina as they are involved in the uptake and degradation of the neurotransmitters glutamate and $\gamma$ aminobutyric acid, in the formation of the blood-retinal barrier and in the provision of glucose to the photoreceptors 
[4]. Signs of Müller cell dysfunction have been found at an early stage of diabetes, before the appearance of visible retinopathy [44]. The role of the Müller cells in glycaemia adaptation may have to be examined using invasive methods, if the underlying mechanistic is to be identified.

In conclusion, this study demonstrated a long-term adaptation phenomenon of the rod photoreceptor retinal visual pathway in response to persistent glycaemia reduction after initiation of CSII. The change in function may reflect an underlying change in metabolism. The methods used in this study may be of value in future studies of early worsening of diabetic retinopathy and the pathogenesis of diabetic retinopathy.

Acknowledgements We wish to thank the Velux Foundation and Lucens for financial support.

Duality of interest G. R. Jackson holds intellectual property associated with the AdaptDx and is an employee of and investor in Apeliotus Vision Science, the manufacturer or the AdaptDx. All other authors declare that there is no duality of interest associated with this manuscript.

\section{References}

1. Diabetes Control and Complications Trial Research Group (1994) Effect of intensive diabetes treatment on the development and progression of long-term complications in adolescents with insulin-dependent diabetes mellitus: Diabetes Control and Complications Trial. J Pediatr 125:177-188

2. Fong DS, Aiello L, Gardner TW et al (2003) Diabetic retinopathy. Diabetes Care 26(Suppl 1):S99-S102

3. Lieth E, Gardner TW, Barber AJ, Antonetti DA (2000) Retinal neurodegeneration: early pathology in diabetes. Clin Experiment Ophthalmol 28:3-8

4. Fletcher EL, Phipps JA, Ward MM, Puthussery T, WilkinsonBerka JL (2007) Neuronal and glial cell abnormality as predictors of progression of diabetic retinopathy. Curr Pharm Des 13:2699 2712

5. Juen S, Kieselbach GF (1990) Electrophysiological changes in juvenile diabetics without retinopathy. Arch Ophthalmol 108:372375

6. Roy MS, Gunkel RD, Podgor MJ (1986) Color vision defects in early diabetic retinopathy. Arch Ophthalmol 104:225-228

7. Sokol S, Moskowitz A, Skarf B, Evans R, Molitch M, Senior B (1985) Contrast sensitivity in diabetics with and without background retinopathy. Arch Ophthalmol 103:51-54

8. Phipps JA, Fletcher EL, Vingrys AJ (2004) Paired-flash identification of rod and cone dysfunction in the diabetic rat. Invest Ophthalmol Vis Sci 45:4592-4600

9. Phipps JA, Yee P, Fletcher EL, Vingrys AJ (2006) Rod photoreceptor dysfunction in diabetes: activation, deactivation, and dark adaptation. Invest Ophthalmol Vis Sci 47:3187-3194

10. Diabetes Control and Complications Trial (1998) Early worsening of diabetic retinopathy in the Diabetes Control and Complications Trial. Arch Ophthalmol 116:874-886

11. Rosenlund EF, Haakens K, Brinchmann-Hansen O, DahlJorgensen K, Hanssen KF (1988) Transient proliferative diabetic retinopathy during intensified insulin treatment. Am J Ophthalmol 105:618-625
12. Davis MD, Fisher MR, Gangnon RE et al (1998) Risk factors for high-risk proliferative diabetic retinopathy and severe visual loss: Early Treatment Diabetic Retinopathy Study Report \#18. Invest Ophthalmol Vis Sci 39:233-252

13. Frost-Larsen K, Christiansen JS, Parving HH (1983) The effect of strict short-term metabolic control on retinal nervous system abnormalities in newly diagnosed type 1 (insulin-dependent) diabetic patients. Diabetologia 24:207-209

14. Dahl-Jorgensen K, Brinchmann-Hansen O, Hanssen KF, Sandvik L, Aagenaes O (1985) Rapid tightening of blood glucose control leads to transient deterioration of retinopathy in insulin dependent diabetes mellitus: the Oslo Study. BMJ (Clin Res Ed) 290:811815

15. The Kroc Collaborative Study Group (1984) Blood glucose control and the evolution of diabetic retinopathy and albuminuria. A preliminary multicenter trial. N Engl J Med 311:365-372

16. Klemp K, Sander B, Brockhoff PB, Vaag A, Lund-Andersen H, Larsen M (2005) The multifocal ERG in diabetic patients without retinopathy during euglycemic clamping. Invest Ophthalmol Vis Sci 46:2620-2626

17. Kurtenbach A, Langrova H, Zrenner E (2000) Multifocal oscillatory potentials in type 1 diabetes without retinopathy. Invest Ophthalmol Vis Sci 41:3234-3241

18. Shimada Y, Li Y, Bearse MA Jr, Sutter EE, Fung W (2001) Assessment of early retinal changes in diabetes using a new multifocal ERG protocol. Br J Ophthalmol 85:414-419

19. Lovasik JV, Spafford MM (1988) An electrophysiological investigation of visual function in juvenile insulin-dependent diabetes mellitus. Am J Optom Physiol Opt 65:236-253

20. Amemiya T (1977) Dark adaptation in diabetics. Ophthalmologica 174:322-326

21. Di Leo MA, Caputo S, Falsini B et al (1992) Nonselective loss of contrast sensitivity in visual system testing in early type I diabetes. Diabetes Care 15:620-625

22. Jackson GR, Edwards JG (2008) A short-duration dark adaptation protocol for assessment of age-related maculopathy. J Ocul Biol Dis Inform 1:7-11

23. McGwin G Jr, Jackson GR, Owsley C (1999) Using nonlinear regression to estimate parameters of dark adaptation. Behav Res Meth Instrum Comput 31:712-717

24. Holfort SK, Jackson GR, Larsen M (2010) Dark adaptation during transient hyperglycemia in type 2 diabetes. Exp Eye Res 91:710 714

25. Marmor MF, Fulton AB, Holder GE, Miyake Y, Brigell M, Bach M (2009) ISCEV standard for full-field clinical electroretinography (2008 update). Doc Ophthalmol 118:69-77

26. Hood DC, Bach M, Brigell M et al (2008) ISCEV guidelines for clinical multifocal electroretinography (2007 edition). Doc Ophthalmol 116:1-11

27. Lauritzen T, Frost-Larsen K, Larsen HW, Deckert T (1983) Effect of 1 year of near-normal blood glucose levels on retinopathy in insulin-dependent diabetics. Lancet 1:200-204

28. Beck-Nielsen H, Richelsen B, Mogensen CE et al (1985) Effect of insulin pump treatment for one year on renal function and retinal morphology in patients with IDDM. Diabetes Care $8: 585-589$

29. Olsen T, Ehlers N, Nielsen CB, Beck-Nielsen H (1985) Diabetic retinopathy after one year of improved metabolic control obtained by continuous subcutaneous insulin infusion (CSII). Acta Ophthalmol (Copenh) 63:315-319

30. Henson DB, North RV (1979) Dark adaptation in diabetes mellitus. Br J Ophthalmol 63:539-541

31. Holopigian K, Seiple W, Lorenzo M, Carr R (1992) A comparison of photopic and scotopic electroretinographic changes in early diabetic retinopathy. Invest Ophthalmol Vis Sci 33:2773-2780 
32. Kim SY, Naoi N, Honda Y (1986) Effect of glucose added to intraocular irrigating solutions on the rabbit electroretinogram in vitro. Doc Ophthalmol 63:131-135

33. Macaluso C, Onoe S, Niemeyer G (1992) Changes in glucose level affect rod function more than cone function in the isolated, perfused cat eye. Invest Ophthalmol Vis Sci 33:2798-2808

34. Dawson WW, Hazariwala K, Karges S (2000) Human photopic response to circulating glucose. Doc Ophthalmol 101:155-163

35. Klemp K, Larsen M, Sander B, Vaag A, Brockhoff PB, LundAndersen H (2004) Effect of short-term hyperglycemia on multifocal electroretinogram in diabetic patients without retinopathy. Invest Ophthalmol Vis Sci 45:3812-3819

36. Holfort SK, Klemp K, Kofoed PK, Sander B, Larsen M (2010) Scotopic electrophysiology of the retina during transient hyperglycemia in type 2 diabetes. Invest Ophthalmol Vis Sci 51:2790 2794

37. Kofoed PK, Sander B, Zubieta-Calleja G, Kessel L, Klemp K, Larsen M (2009) The effect of high- to low-altitude adaptation on the multifocal electroretinogram. Invest Ophthalmol Vis Sci 50:3964-3969
38. Birol G, Wang S, Budzynski E, Wangsa-Wirawan ND, Linsenmeier RA (2007) Oxygen distribution and consumption in the macaque retina. Am J Physiol Heart Circ Physiol 293:H1696-H1704

39. Linsenmeier RA (1986) Effects of light and darkness on oxygen distribution and consumption in the cat retina. J Gen Physiol 88:521-542

40. Birol G, Budzynski E, Wangsa-Wirawan ND, Linsenmeier RA (2005) Retinal arterial occlusion leads to acidosis in the cat. Exp Eye Res 80:527-533

41. Arden GB, Wolf JE, Tsang Y (1998) Does dark adaptation exacerbate diabetic retinopathy? Evidence and a linking hypothesis. Vis Res 38:1723-1729

42. Yau KW, Baylor DA (1989) Cyclic GMP-activated conductance of retinal photoreceptor cells. Annu Rev Neurosci 12:289-327

43. Hood DC, Birch DG (1996) Beta wave of the scotopic (rod) electroretinogram as a measure of the activity of human on-bipolar cells. J Opt Soc Am A Opt Image Sci Vis 13:623-633

44. Lieth E, Barber AJ, Xu B et al (1998) Glial reactivity and impaired glutamate metabolism in short-term experimental diabetic retinopathy. Penn State Retina Research Group Diabetes 47:815-820 